\title{
Gluonic fields as unraveled with Polyakov lines and predicted by bosonic strings
}

\author{
Ahmed S. Bakry ${ }^{* \dagger}$ \\ Adelaide University, SA, 5005 \\ E-mail: abakry@Physics.adelaide.edu.au
}

Derek Leinweber

Adelaide University, SA, 5005

\section{Anthony Williams}

Adelaide University, SA, 5005

\begin{abstract}
The gluonic action density has been revealed in baryons and mesons using Polyakov loops as a quark source operator at finite temperature. The action density in the baryon exhibits a filled $\Delta$-shaped profile which persists even at large quark separation. The density distribution is nonuniform with a maximum localized near the Fermat point of the quark configuration. The mesonic action density profile is non-uniform and curved. The width of the flux tube is non-constant along the line joining the quark-antiquark at large distance for the temperature just close to the deconfinement point. The width profile of the action density of both systems is consistent with the predictions of both the mesonic and the Y-shaped baryonic string models predictions.
\end{abstract}

The 30 International Symposium on Lattice Field Theory - Lattice 2012,

June 24-29, 2012

Cairns, Australia

* Speaker. 


\section{Introduction}

The confinement of quarks into mesons and hadrons is an outstanding feature of QCD, the theory of strong interactions. Computer simulations have revealed that quark confinement is a property of the gluonic sector and is common for non-Abelian gauge theories. In fact, the potential of a static quark anti-quark pair linearly rises with the inter-quark distance. The origin of the linearly rise has been identified to be due to colour-electric flux tube which forms between the quark colour sources. The flux tube has been made visible in zero-temperature SU(3) Yang-Mills theory or quark anti-quark (mesonic) and three quark (baryonic) configurations by studying the correlations between field strength and 'Polyakov lines' and 'Wilson loops', respectively. Investigating the questions why the colour-electric flux tube forms is an active field of research.

It is well known that Yang-Mills theories at high temperatures cease to confine quarks: the static quark potential with its linearly rising asymptotic form turns into a Coulomb type potential if the temperature exceeds a critical value. The properties of the confining flux tubes close but below the critical temperature are hitherto unknown. The focus of this research has been on re-addressing the colour-electromagnetic flux tube in mesonic and baryonic configurations by extending the existing considerations to finite temperatures.
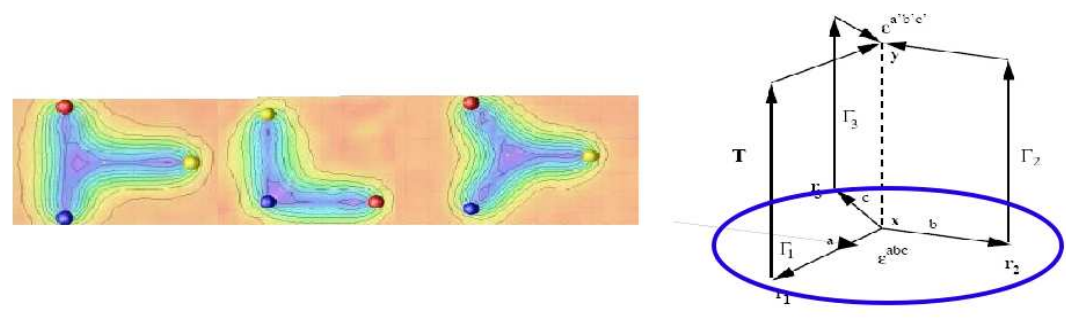

Figure 1: (Right) 3Q Wilson loop, for a fixed source geometry, many different spatial-links configurations are possible. (Left) Dependence of the flux density on the choice of the source operator indicates that non-ground state contamination contribute to the correlation function Ref. [1].

Technically, this involves evaluating correlations of Polyakov lines with a field strength operator. The study is demanding and challenging from the numerical point of view as it discusses correlations involving the ratio of two exponentially decaying operators. The use of a set of unbiased operators to reveal the field structure within nucleons has also been an attractive idea to overcome the systematic errors associated with utilizing the overlap formalism of Wilson operators. In addition, these lattice calculations are necessary to look into and identify the signatures of the confining bosonic strings unambiguously in QCD.

Besides overcoming the systematic errors associated with the Wilson loop approach (see Fig. [1]), the objectives of this investigation focused on revealing and appreciating the changes on the energy distribution with the temperature based on string models as well. The aim of the present proceeding is to present a summary of the work that has accomplished in the study of the gluonic structure in the mesonic and baryonic configurations from the above mentioned perspective. 

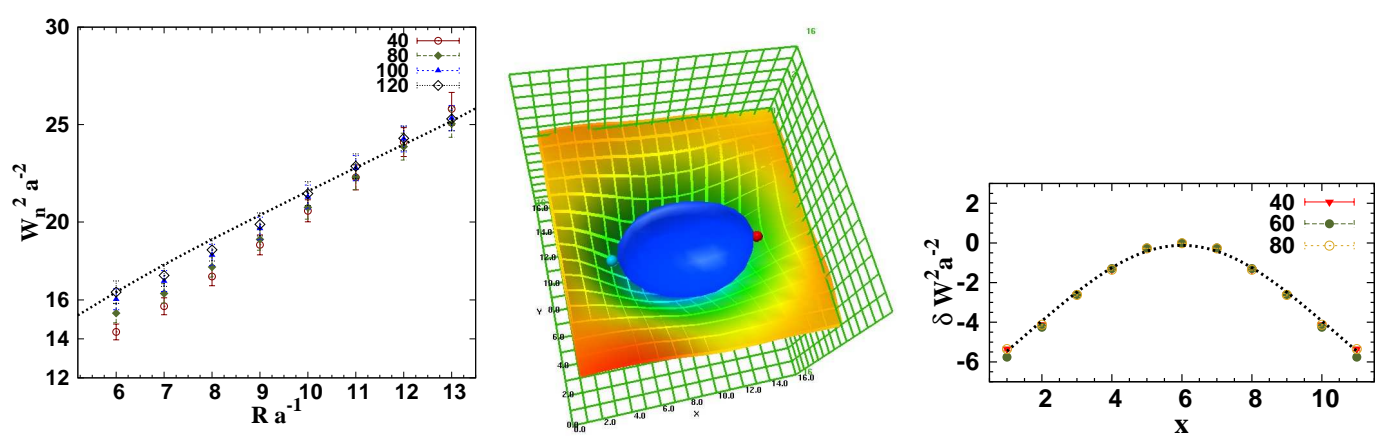

Figure 2: (Left) The flux-tube's width in the middle plane versus quark source separation for various levels of UV filtering. (Middle) The action density iso-surface for quark source separation $\mathrm{d}=0.9 \mathrm{fm}$ and temperature $\mathrm{T}=0.8 \mathrm{~T}_{\mathrm{C}}$. (Right) The change of the tube's width $\delta W^{2}=W^{2}\left(x_{i}\right)-W^{2}\left(x_{0}\right)$ measured from the central plane for the depicted $q \bar{q}$ separations $\mathrm{d}=1.2 \mathrm{fm} \beta=6, \mathrm{~T}=0.9 \mathrm{~T}_{\mathrm{C}}$. The line denotes the width difference $\delta W^{2}$ as predicted by the string model.

The first contribution to this point has been reported in Ref. [2], where the ideas learned from bosonic string models and noise reduction techniques have been applied to the problem of investigating gluonic fields of the meson in pure $\mathrm{SU}(3)$ gauge theory at finite temperature below the deconfinement point. The profile of the confining flux tubes has been revealed in the meson for two temperatures below the deconfinement point and then compared to the predictions from bosonic string models. The work discusses the changes on the whole profile of the flux tubes due to the temperature based on bosonic string models. The width profile of the revealed flux-tubes is curved and linearly growing (see Fig. [2]) near the deconfinement point, which is consistent with the string picture predictions at large distances.

As a continuation of this analysis, the effect of filtering out ultraviolet fluctuations have been investigated and compared with string model predictions in Ref. [3]. It has been shown that the broadening properties of the free bosonic strings are independent of the UV physics. The following items summarise the main results in the two Refs. $[2,3]$ mentioned above:

- Unlike the gluonic profile displayed at zero temperature using Wilson loops as a quark source operator, the profile of the flux-tube action density displays a prolate-spheroid-like shape. This result is obtained after correlating two Polyakov loops as quark source operators with an action density [2].

- At large distances, the flux tube exhibits a non-constant width profile consistent with the predictions of the bosonic string model at finite temperatures and the action density is nonuniformly distributed [2].

- Verification of the linear growth of the flux tube width near the deconfinement point with the increase of the inter-quark separation in pure $\mathrm{SU}(3)$ gauge theory $[2,3]$.

- The effective string physics is independent from the ultraviolet filtering of the vacuum fluctuations [3].

- The ultraviolet filtering of short distance vacuum fluctuations reveals the stringy properties of the flux tube at intermediate inter-quark separations at high temperatures [3]. 

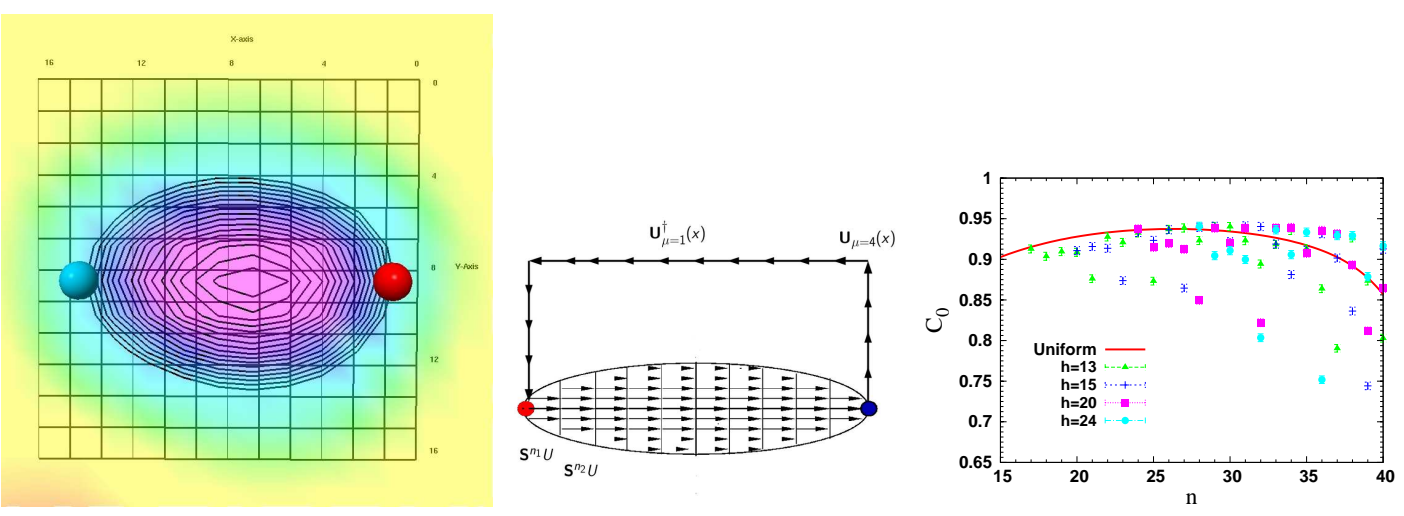

Figure 3: (Left) Action isolines in the quark-antiquark plane for temperature $T=0.8 T_{c}$ near the end of QCD plateau for quark separation $d=1 \mathrm{fm}$.(Middle) Trial states with a non-uniform smearing profile of the spatial links of Wilson loops have been found to optimize the overlap with the mesonic ground state. (Right) Measurement of the overlap of the ground state meson potential for elliptical source shapes parametrized with number of sweeps $h$ (at the last spatial link) and $n$ (at the middle spatial link). The continuous line denote the overlap of uniformly smeared or (flat) states $n=h$.

Figure [2] illustrates the above mentioned points.

Some of the 'non-uniformly' distributed gluonic states revealed at finite temperature acquires a constant width (in particular those at the end of the QCD Plateau). This observation has motivated the importance of investigating the trial states corresponding to non-uniform gluonic distributions, in obtaining the ground state of the theory.

At zero temperature, the overlap of trial states, created via space-dependent levels of APE smearing with the ground state, is extracted using the transfer formalism (see Fig. [3]). This has been reported in Ref. [4]. The conclusion of the investigation, is that a non-uniform flux tube (similar to the finite temperature case) possesses a higher overlap with the true ground state. This could imply that the ground state flux tube is curved rather than straight and would have greater suppression of the QCD action density in the centre of the flux tube. The result motivates, extending the investigations with Polyakov lines into lower temperatures.

The study has been extended to the baryonic flux tube arrangement at finite temperature using Polyakov lines and cooling for noise reduction. The interesting result is that the gluonic flux displays, a profile consistent with a filled $\Delta$-shape, even at large distance which seems to contrast the famed Y-shaped flux tube obtained via Wilson-loops correlations at low temperatures. This baryonic flux tube system has been analysed in detail from various aspects of the profile in Ref. [5].

The profile of the baryonic string has been unambiguously identified directly via fit behaviour of a form consisting of a sum of two-Gaussians. The Y-baryonic string model has been discussed at high temperature for the Width profile of the junction in Refs. $[6,7,8]$. The lattice data for the mean-square width of the gluonic action density has been compared to the corresponding width calculated based on string model at finite temperature. The best fits for the string model are returned for large quark source separation only assuming the classical position of the strings at the Fermat point of the configuration (point that minimizes the length of Y-strings). This analysis [7] can be of a particular relevance to the confining string models, as it is the first time we can directly look and identify a clear formation of Y-shaped confining strings in the baryonic filled $\Delta$-shaped action 
density, from the first principles of QCD. In Ref. [7] primary numerical indications have been also summarised.
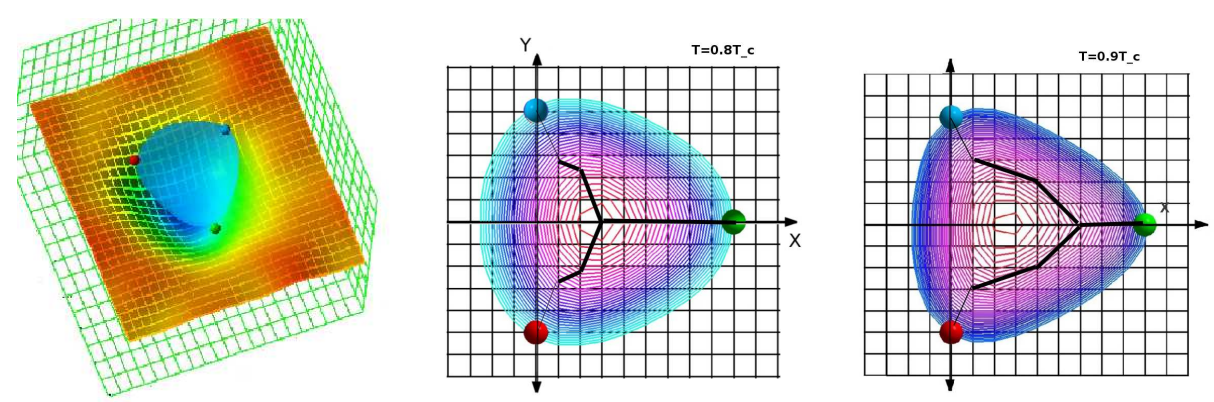

Figure 4: (Left) The action density iso-surface is a filled $\Delta$-shape in the baryon for temperature $T_{c}=0.8$ for a 3Q configuration of isosceles base length $A=1.0 \mathrm{fm}$ and height of roughly $R=1.0 \mathrm{fm}$. (Middle) The action isolines in the $3 \mathrm{Q}$ plane for temperature at the end of the QCD plateau region $T=0.8 T_{c}$. The lines superimposed on the density plot refer to the position of the center of each of the two overlapping Gaussians used to fit the action density in the transverse planes to the $\mathrm{x}$-axis. In the confining string model, these lines indicate the mean position of the gluonic strings joining the $3 \mathrm{Q}$ system. (Right) Same as the adjacent plot but for the temperature just before deconfinement $T=0.9 T_{c}$. This plot shows that the notable change on the profile of the baryonic flux arrangement, with the temperature increase, is the movement of the junction to the inner region of the quark configuration.
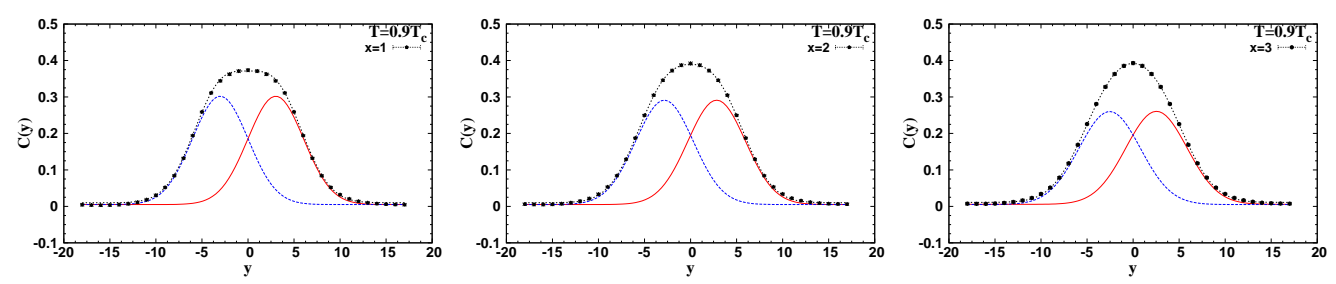

Figure 5: The density distribution $\mathscr{C}^{\prime}(\vec{\rho})$ for the isosceles configuration with the base, $A=1.0 \mathrm{fm}$, and height $R=0.7 \mathrm{fm}$ at $\left.T / T_{c}=0.9\right)$. Data are plotted for the transverse planes $x=1$ to $x=3$. The lines correspond to fits of the sum of two Gaussians fits to the density in each plane $\vec{\rho}\left(x_{i}, y, 0\right)$. The distance between the two Gaussians gets closer which ultimately coincide at 'a junction' as we move into farther planes from the base of the triangle.

The following summarises the main results of the baryonic analysis:

- The gluonic action-density exhibits a filled- $\Delta$-shaped profile even at large quark separation [5, 7] (See Fig. [4]).

- An underlying Y-shaped flux tube system, for the $\Delta$-shaped action is revealed through a double Gaussian fits [8] (see Figs. [4, 5]). This result might indicate that the Y-shaped flux arrangement is a rough approximation to the ground state energy density.

- Verifying the validity of the Y-baryonic string model at finite temperatures. This shows that the $\Delta$-shaped gluonic profile comes about, through the vibration of the underlying Y-string system $[7,8]$. 
- The lattice data for the baryonic flux tubes near the deconfinement point indicates a linear growth pattern for the baryonic flux-tube [5].

Investigating the energy distribution within elementary particle systems is a fundamental subject to QCD and confinement. Re-addressing the confining flux tubes at finite temperature scales is an interesting topic not only for the understanding of the changes on the profile of the flux-tube with the temperature, but also for the reason that the revealed color map in baryons and mesons with Polyakov loops is presenting a potential candidate for the exact geometry of the flux tubes arrangements, if the analysis with Polyakov loops is to be extrapolated to zero temperatures.

\section{References}

[1] F. Bissey, et al, "Gluon flux-tube distribution and linear confinement in baryons," Phys. Rev. D 76, 114512 (2007) [hep-lat/0606016].

[2] A. S. Bakry, et al, "String effects and the distribution of the glue in mesons at finite temperature," Phys. Rev. D 82, 094503 (2010) [arXiv:1004.0782 [hep-lat]].

[3] A. S. Bakry, D. B. Leinweber and A. G. Williams, "Bosonic stringlike behavior and the Ultraviolet filtering of QCD," Phys. Rev. D 85, 034504 (2012) [arXiv:1011.1380 [hep-lat]].

[4] A. S. Bakry, D. B. Leinweber and A. G. Williams, "On the ground state of Yang-Mills theory," Annals Phys. 326, 2165 (2011) [arXiv:1102.3477 [hep-lat]].

[5] A. S. Bakry, D. B. Leinweber and A. G. Williams, "Gluonic profile of the static baryon at finite temperature," [arXiv:1107.0150 [hep-lat]].

[6] A. S. Bakry, D. B. Leinweber and A. G. Williams, "The thermal delocalization of the flux tubes in mesons and baryons," AIP Conf. Proc. 1354, 178 (2011).

[7] A. S. Bakry, D. B. Leinweber and A. G. Williams, "Gluonic profile of the static baryon at finite temperature and Y-baryonic string," PoS LATTICE 2011, 256 (2011)

[8] (To appear), A. S. Bakry, D. B. Leinweber and A. G. Williams, "A look at the confining Y-baryonic strings in the baryonic $\Delta$-flux" ADP-11-16/T738. 